\title{
Environmental influence on the genetic correlations between life-history traits in Caenorhabditis elegans
}

\author{
EW Gutteling, A Doroszuk, JAG Riksen, Z Prokop, J Reszka and JE Kammenga \\ Laboratory of Nematology, Wageningen University, Wageningen, The Netherlands
}

\begin{abstract}
Empirical evidence is mounting to suggesting that genetic correlations between life-history traits are environment specific. However, detailed knowledge about the loci underlying genetic correlations in different environments is scant. Here, we studied the influence of temperature $\left(12^{\circ} \mathrm{C}\right.$ and $24^{\circ} \mathrm{C}$ ) on the genetic correlations between egg size, egg number and body mass in the nematode Caenorhabditis elegans. We used a quantitative trait loci (QTL) approach based on a genetic map with evenly spaced single nucleotide polymorphism markers in an $\mathrm{N} 2 \times$ CB4856 recombinant inbred panel. Significant genetic correlations between
\end{abstract}

various traits were found at both temperatures. We detected pleiotropic or closely linked QTL, which supported the negative correlation between egg size and egg number at $12^{\circ} \mathrm{C}$, the positive correlation across temperatures for body mass, and the positive correlation between body mass and egg size at $12^{\circ} \mathrm{C}$. The results indicate that specific loci control the covariation in these life-history traits and the locus control is prone to environmental conditions.

Heredity (2007) 98, 206-213. doi:10.1038/sj.hdy.6800929; published online 3 January 2007

Keywords: Caenorhabditis elegans; life history trait; quantitative trait loci mapping; pleiotropy; genetic correlation

\section{Introduction}

Over the last decades there has been an increased awareness that environmental conditions can influence the genetic control of life-history traits. Empirical evidence is mounting up suggesting that genetic correlations, those correlations that arise because genes affect multiple traits, are environment specific. It has been shown that these correlations can even switch signs (positive vs negative) across environments (Sgrò and Hoffmann, 2004). For instance Czesak and Fox (2003) found that the sign of the genetic correlation between egg size and female body mass in the seed beetle Stator limbatus depended on the type of food consumed. Hausmann et al. (2005) studied the influence of water deficit on the genetic correlation among life-history traits in Arabidopsis thaliana. Early-flowering plants produced more fruits in well-watered treatments, whereas no such correlation existed under water-limited conditions. They also reported that the genetic correlations of fruit number with branch number and flowering time were not symmetric. Fruit number in the wet environment was positively correlated with number of branches in the dry environment and negatively correlated with flowering time in the dry environment, whereas fruit number in the dry environment was not correlated with either trait in the wet environment (Hausmann et al., 2005).

Correspondence: Dr JE kammenga, Laboratory of Nematology, Wageningen University, Binnenhaven 5, 6709 PD Wageningen, The Netherlands. E-mail: Jan.Kammenga@wur.nl

Received 7 March 2006; revised 6 July 2006; accepted 23 November 2006; published online 3 January 2007
If genetic correlations between life-history traits depend on environmental conditions, then this has major implications for the evolutionary trajectories of these traits, especially in the case where genetic correlations are negative and reflect trade-offs between traits. Tradeoffs between life-history traits have been a well studied subject in evolutionary biology (Stearns, 1992; Roff, 2000). In particular the trade-off between egg size and number which results from genetic constraints (Winkler and Wallin, 1987; Roff, 2000) has been well documented (Fox, 1997; Vaughton and Ramsey, 1998).

Negative genetic correlations suggest the existence of loci with pleiotropic or closely linked effects. For example, senescence has been explained as the antagonistic pleiotropic effect of genes that are beneficial early in life but deleterious later in life (Williams, 1957). Evidence that such genes indeed exist has come from the field of molecular genetics. In the nematode Caenorhabditis elegans specific mutations in the genes daf-2 (Gems et al., 1998) or clk-1 (Hekimi et al., 1998) increase lifespan by a factor of two while simultaneously decreasing fecundity by an equal factor. On the other hand, Kenyon et al. (1993) showed than another daf-2 mutation decoupled reproduction from lifespan. It should be realized however that artificial mutations with a large effect, both on monogenic as polygenic traits, are rare in nature. As pointed out by Knight et al. (2001), these mutations do not necessarily reveal information on the segregating alleles that are selected for during evolution and that contribute to fitness in natural populations.

Instead, such alleles could be identified using quantitative trait loci (QTL) mapping. This approach has been used to unravel the genetic basis of genetically correlated 
traits in various species. Hausmann et al. (2005) reported that the negative genetic correlations in A. thaliana, both between fruit length and fruit production and between flowering time and branch production, were corroborated by pleiotropic or closely linked QTL. Juenger et al. (2005) also used a QTL mapping approach for studying the genetic basis of correlated floral and leaf morphology traits in $A$. thaliana. They found large positive genetic correlations among flower and leaf traits that were supported by linked QTL.

The nematode $C$. elegans is an important model for genetic mapping of life-history traits because of its ease of handling and observation, short generation time, and completely sequenced genome. Shook and Johnson (1999) studied QTL affecting survival and fertilityrelated traits and found genotype-environment interactions and pleiotropy for a number of traits. Ayyadevara et al. (2003) studied the genetic loci modulating fitness and life span in C. elegans. Allelic longevity effects for two QTL, on chromosomes $I V$ and $V$, were confirmed in backcrossed congenic lines and were highly significant in two very different environments - growth on solid agar medium and in liquid suspension culture. Yet, only one study by Knight et al. (2001) has focused on the correlations (within one environment) and trade-offs between life-history traits (egg number and body mass). They found that a QTL for egg number colocalized with the body mass QTLs on chromosome IV suggesting pleiotropic or closely linked effects.

Over the last decade $C$. elegans has become a model system for studying gene-environment interactions such as behavioral responses to food density, chemotaxis and thermotaxis using mutational mapping approaches. A few phenotypic studies showed that various traits were strongly influenced by temperature change (Byerly et al., 1976; Van Voorhies, 1996) and suggested a correlation between body mass, egg number and egg size.

Here, we studied the genetic control of these lifehistory traits at two different temperatures using QTL mapping in C. elegans recombinant inbred lines (RILs). We have exposed the RILs to $12^{\circ} \mathrm{C}$ and $24^{\circ} \mathrm{C}$, temperatures that strongly affect egg size and number. We first sought to determine the effect of temperature on genetic correlations between egg size, egg number and body mass. We then aimed at determining the genomic regions associated with these correlations (at $12^{\circ} \mathrm{C}$ and $24^{\circ} \mathrm{C}$ ). The existence of colocalizing QTL within temperatures might suggest pleiotropic effects or close linkage. Colocalizing QTL across temperatures would suggest a similar genetic control of traits under different temperature conditions.

\section{Materials and methods}

Nematode culturing, construction of RILs and description of the genetic map

Both N2 and CB4856 parental strains were homozygous. These strains were grown in $9-\mathrm{cm}$ Petri-dishes at $15^{\circ} \mathrm{C}$ or $20^{\circ} \mathrm{C}$ on standard nematode growth medium with Escherichia coli strain OP50 as food source (Lewis and Fleming, 1995), and transferred to new dishes by a chunk of agar once a week. RILs (see Li et al. (2006)) were constructed by putting on each of ten $6 \mathrm{~cm}$ dishes, one $\mathrm{J} 4$ hermaphrodite of strain N2 with five males of strain CB4856, and vice versa on each of 10 other $6 \mathrm{~cm}$ dishes to avoid any maternal or paternal effects. Mating was considered to be successful if the ratio of males:hermaphrodites was approximately $1: 1$ in the F1 hybrids. Approximately 1500 F1 hermaphrodites were transferred to individual dishes in 24-well plates and allowed to selffertilize at $20^{\circ} \mathrm{C}$. This procedure was repeated until the F20.

After inbreeding, RILs were stored at $-80^{\circ} \mathrm{C}$ using standard protocols (Lewis and Fleming, 1995) before being used in experiments. Using the C. elegans SNP website (http://genome.wustl.edu/projects/celegans/ index.php?snp =1) we selected 20 markers for chromosomes $I, I I, I I I, I V$ and $X$, and 21 for chromosome $V$ (because of its larger physical size). QTL mapping was based on a genome wide single nucleotide polymorphism (SNP) map consisting of the 121 markers. The average distance between two markers was $835 \mathrm{kbp}$ or $2.38 \mathrm{cM}$ with a genome-wide coverage of $95.9 \%$ if measured in bp or $95.3 \%$ if measured in cM. Details on the RILs can be found in Li et al. (2006).

\section{Phenotypic analysis of parents and RILs}

Before being used in experiments, parents and RILs were cultured at $15^{\circ} \mathrm{C}$ for at least 4 weeks after recovery from $-80^{\circ} \mathrm{C}$ storage. Each parental strain and the 80 RILs were analyzed for egg size, egg number and body mass at $12^{\circ} \mathrm{C}$ and $24^{\circ} \mathrm{C}$ as described below. All experiments were carried out in two Elbanton climate chambers (Elbanton, $\mathrm{NL}$, USA). One chamber was set at $12^{\circ} \mathrm{C}$, the other at $24^{\circ} \mathrm{C}$. Temperature was monitored with a Tinytag Transit temperature logger (Gemini Data Loggers, UK). The following procedure was used, following Gutteling et al. (2007).

Egg number and egg size: RILs were synchronized by bleaching (Emmons et al., 1979). Bleached eggs from each RIL were divided over four Petri-dishes of which two were put at $12^{\circ} \mathrm{C}$ and two at $24^{\circ} \mathrm{C}$. From each temperature, 14 L4 juveniles from each RIL were randomly chosen from the two dishes and individually transferred to separate dishes and subsequently allowed to mature at $24^{\circ} \mathrm{C}$ or $12^{\circ} \mathrm{C}$. After maturation adults were transferred daily $\left(24^{\circ} \mathrm{C}\right)$ or every four days $\left(12^{\circ} \mathrm{C}\right)$ (because $C$. elegans develops and reproduces faster at high temperatures and therefore food will be depleted sooner at $24^{\circ} \mathrm{C}$ than at $12^{\circ} \mathrm{C}$ ) to new dishes until the worms stopped laying eggs. Egg number was recorded as total number of eggs produced by a single worm.

Pictures were taken from the eggs with a CoolSnap camera (Roper Scientific Photometrics, Tucson, AZ, USA) at $\times 43.75$ magnification. The area and perimeter of each egg were measured automatically with Image Pro Express 4.0 (Media Cybernetics, Silver Spring, MD, USA). Using a measurement ocular, we calibrated 10.000 pixels $^{3}$ as $7591 \mu \mathrm{m}^{3}$. Using an estimated radius $r$ (in pixels) for each egg, we calculated the volume $V_{E G G}$ with the volume sphere function:

$$
V_{E G G}=\frac{4}{3} \pi r^{3}
$$

In subsequent analyses $V_{E G G}$ was used as input value for egg size.

Body mass: Body mass was defined as the body mass at maturation. RILs were synchronized by $2.5 \mathrm{~h}$ of egg 
laying by four gravid adults. For each replicate 12 juvenile nematodes were individually transferred at room temperature to separate $3 \mathrm{~cm}$ Petri-dishes. After transferring all worms in one replicate experiment dishes were randomized within the replicate and returned to the appropriate temperature. Analyzing individuals for maturation started after $38 \mathrm{~h}$ for $24^{\circ} \mathrm{C}$ and after $145 \mathrm{~h}$ for $12^{\circ} \mathrm{C}$. Dishes were analyzed at intervals of $1.5 \mathrm{~h}$ for $24^{\circ} \mathrm{C}$ and $4 \mathrm{~h}$ for $12^{\circ} \mathrm{C}$. Maturation was defined as the first moment at which one or more laid eggs were visually observed on a dish. If so, the time of observation was registered and the dish was put at $-20^{\circ} \mathrm{C}$ to prevent further development of the nematode. Dishes were defrosted and dead bodies were transferred to new dishes with agar to prevent dehydration of the body. A pilot study (data not shown) had shown that freezing did not affect body size. Digital pictures were taken of the bodies at $\times 7.25$ magnification. Using a measurement ocular we calibrated 10.000 pixels $^{3}$ as $753.516 \mu \mathrm{m}^{3}$. Area $\left(A\right.$, pixels $\left.{ }^{2}\right)$ and perimeter $(P$, pixels) of each worm were measured digitally (see above for hardware used). Assuming that the body of a nematode resembles a cylinder these values were converted to the volume $V_{S Z M}$ using the function:

$$
V_{S Z M}=\frac{\pi A^{2}}{2 P}
$$

In subsequent analyses $V_{S Z M}$ was used as input value for body mass.

\section{Statistics and computations}

All data were tested for normality using a one-sample Kolmogorov-Smirnov test. The effect of temperature on egg number, egg size and body mass for the parents were anlaysed with a $t$-test and one-way ANOVA. For both temperatures mean values of body mass, egg number and egg size were computed for each RIL to be used in QTL analysis. Only mean trait values based on data of more than nine individuals were used. The influence of temperature, RIL and their interaction were tested using PROC GLM (SAS, 2003). Broad-sense heritabilities $\left(\mathrm{H}^{2}\right)$ and s.e. were computed according to Phillips and Arnold (1999). Variances in the traits were calculated from variance components using the restricted maximum likelihood method (SAS PROC VARCOMP) (SAS, 2003). Genetic correlations between traits $i$ and $j$ were estimated as $\operatorname{cov}(i, j) / \sigma_{i} \sigma_{j}$, where $\operatorname{cov}(i, j)$ is the covariance among RILs means for $i$ and $j$, and $\sigma_{i}$ and $\sigma_{j}$ are the s.d. of these traits. The significance of the correlation within each temperature was determined using a $t$-test after a $z$-transformation of the correlation coefficients (Sokal and Rohlf, 1995). Test of homogeneity between correlation coefficients $(r)$ and the comparison between the slopes $(b)$ in two environments were performed with $t$ statistic according to Sokal and Rohlf (1995).

\section{QTL mapping}

QTL mapping was performed with QTL Cartographer (Wang et al., 2001). We first analyzed all traits by interval mapping (IM) to detect any strong QTL. Subsequently we analyzed the data by composite interval mapping (CIM) assuming multilocus control of the trait. Settings during the analyses for both analyses were default (walk speed $2 \mathrm{cM}$, model number 6,5 background parameters, window size $10 \mathrm{cM}$, and automatic selection of the background parameters). The experiment-wise threshold significance level for each trait was determined by performing 10000 permutations on the data (Churchill and Doerge, 1994). 95\% confidence intervals for QTL were calculated according to Dupuis and Siegmund (1999).

The significance of pairwise epistatic interactions between QTL was evaluated by mixed linear modeling approach (Wang et al., 1999). The genotype of each marker closest to each significant QTL peak was used to assess the significance on the following interactions: egg size $\times$ number, egg number $\times$ body mass, egg size $\times$ body mass. Although weak interactions were found for only a few marker pairs, they did not include the main-effect QTLs detected by composite interval mapping at $P=0.05$.

\section{Results}

Table 1 shows the phenotypic values and quantitative genetic parameters for egg size and number and body mass for parental and RILs in C. elegans at $12^{\circ} \mathrm{C}$ and $24^{\circ} \mathrm{C}$. Egg number in strain N2 was significantly higher at $12^{\circ} \mathrm{C}$ than at $24^{\circ} \mathrm{C}$ ( $t$-test, $P=0.003$ ) while there was no difference in egg number in CB4856 between these temperatures. With regard to egg size, the parental strains response was significantly different. Egg sizes from CB4856 decreased with $21.3 \%$ in size in response to higher temperatures while N2 egg sizes decreased only with $8.3 \%$ analysis of variance (ANOVA, $p_{\mathrm{GxE}}<0.001$, $\left.p_{\mathrm{E}}<0.001\right)$. With regard to body mass parental strains responded differently to the environment (ANOVA, significant effect of temperature, $P=0.04$ ). N2 grew larger at $12^{\circ} \mathrm{C}$ than $\mathrm{CB} 4856$. At both temperatures $\mathrm{N} 2$ worms were significantly bigger than CB4856 individuals (ANOVA, significant effect of strain at $P<0.001$ ).

Mean trait values were normally distributed (data not shown). Both temperature, RIL and their interaction significantly affected egg size and egg number (ANOVA, $P<0.001)$. For body mass we also found significant effects of temperature, RIL and their interaction $(P<0.001$ for all effects).

\section{Genetic correlations within and across temperatures}

Within temperatures: At $12^{\circ} \mathrm{C}$ we found a significant negative genetic correlation $(P<0.05)$ between egg size and number (Table 2 ) suggesting that the production of more eggs comes at the expense of smaller eggs. Body mass tended to be correlated positively with egg size. At $24^{\circ} \mathrm{C}$, the correlation between egg size and number was not significant, instead we found a significant positive correlation between egg size and body mass.

Across temperatures: The genetic correlation across temperatures considers one trait measured in the two temperatures as two traits, and its magnitude signifies the degree to which the trait is under the same genetic control in each environment. We found significant positive correlations for egg size and body mass indicating a genetic dependence across temperatures. However, we did not detect a correlation for egg number, which suggests that the genetic control for this trait differs for the two temperatures. Body mass at $24^{\circ} \mathrm{C}$ was positively correlated with egg size at $12^{\circ} \mathrm{C}$ whereas body 
Table 1 Phenotypic values and heritabilities $\left(\mathrm{H}^{2}\right)$ for life-history traits in parental strains and RILs

\begin{tabular}{|c|c|c|c|c|c|}
\hline \multirow[t]{2}{*}{ Trait } & \multirow[t]{2}{*}{ Strain } & \multicolumn{2}{|c|}{ Mean \pm s.d. } & \multicolumn{2}{|c|}{$H^{2}$} \\
\hline & & $12^{\circ} \mathrm{C}$ & $24^{\circ} \mathrm{C}$ & $12^{\circ} \mathrm{C}$ & $24^{\circ} \mathrm{C}$ \\
\hline \multirow[t]{3}{*}{ Body mass } & N2 & $1.9(0.1)^{*}$ & $1.8(0.2)$ & & \\
\hline & CB4856 & $1.5(0.2)$ & $1.5(0.2)$ & & \\
\hline & RI strains & $1.9(0.1)^{* * * *}$ & $1.7(0.1)$ & $0.65(0.06)$ & $0.39(0.04)$ \\
\hline \multirow[t]{3}{*}{ Egg number } & N2 & $234.3(13.4)^{*}$ & $177.4(10.4)$ & & \\
\hline & CB4856 & 159.9 (16.9) & $156.6(12.8)$ & & \\
\hline & RI strains & $172.7(72.0)^{*}$ & $176.6(62.4)$ & $0.19(0.03)$ & $0.21(0.03)$ \\
\hline \multirow[t]{3}{*}{ Egg size } & N2 & $3.4(0.3)^{*}$ & $3.1(0.4)$ & & \\
\hline & CB4856 & $3.8(0.4)^{*}$ & $3.0(0.5)$ & & \\
\hline & RI strains & $3.5(0.4)^{*}$ & $2.9(0.4)$ & $0.37(0.04)$ & $0.29(0.03)$ \\
\hline
\end{tabular}

Body mass in $10^{6} \mu \mathrm{m}^{3}$, egg size in $10^{4} \mu \mathrm{m}^{3}$. s.d. in brackets. Significantly different between temperatures: ${ }^{*} P<0.05,{ }^{* * *} P<0.001$, s.e. of $\mathrm{H}^{2}$ in brackets.

Table 2 Genetic correlations ( $r$ ) among the measured life-history traits within temperatures (a) and across temperatures (b)

\begin{tabular}{|c|c|c|c|}
\hline & Egg size & Egg number & Body mass \\
\hline \multicolumn{4}{|c|}{ (a) Within temperatures } \\
\hline \multicolumn{4}{|c|}{ Fog size } \\
\hline \multirow{3}{*}{$\begin{array}{l}\text { Egg number } \\
\text { Body mass }\end{array}$} & $0.07^{24}$ & & $0.05^{12}$ \\
\hline & $0.23(0.02)^{24}$ & $0.05^{24}$ & \\
\hline & Egg size $e^{12}$ & Egg number ${ }^{12}$ & Body mass ${ }^{12}$ \\
\hline \multicolumn{4}{|c|}{ (b) Across temperatures } \\
\hline Egg size $^{24}$ & $0.52(0.02)$ & -0.1 & 0.14 \\
\hline Egg number ${ }^{24}$ & -0.03 & 0.02 & $0.3(0.02)$ \\
\hline Body mass ${ }^{24}$ & $0.46(0.02)$ & -0.08 & $0.49(0.02)$ \\
\hline
\end{tabular}

Superscripts refer to the respective temperatures $\left(12^{\circ} \mathrm{C}\right.$ and $\left.24^{\circ} \mathrm{C}\right)$. Significant correlations $(P<0.05)$ are shown in bold (see text for further information). 95\% confidence intervals calculated as $r \pm 1.96\left(1-r^{2}\right) / \sqrt{ }(n-3)$ (where $n=$ number of samples) between brackets.

mass at $12^{\circ} \mathrm{C}$ was positively correlated with egg number at $24^{\circ} \mathrm{C}$.

There was insufficient evidence to reject the null hypothesis of homogeneity of correlation coefficients and slopes between the temperatures for egg size vs number $\left(t_{\infty}=1.16, P=0.25\right.$ and $t_{151}=1.31, P=0.19$; for $r$ and $b$ respectively). Similarly, neither egg size and body mass $\left(t_{\infty}=0.48, P=0.63\right.$ and $t_{151}=0.27, P=0.79$, for $r$ and $b$, respectively) nor egg number and body size $\left(t_{\infty}=0.40, P=0.69\right.$ and $t_{152}=0.36, P=0.72$, for $r$ and $b$, respectively) showed significant differences between temperatures.

To gain further insight into the genetic control of the significant correlations we conducted QTL analysis at both temperatures. We hypothesized that genetic correlations (which were all found to be all less than one) should be supported by the detection of pleiotropic or closely linked loci.

\section{QTL analyses}

Figure 1 shows the QTL patterns for egg size and number and body mass at $12^{\circ} \mathrm{C}$ and $24^{\circ} \mathrm{C}$. QTL peaks that appeared after IM also appeared in the CIM analyses. Therefore, only the results of the CIM procedure are shown. Permutation-based threshold values for both traits were more or less equal at one temperature; consequently only one threshold is shown in each figure.
We used at both temperatures the most conservative $95 \%$ threshold (i.e., the threshold with the highest value), matching $2.71 \mathrm{LOD}$ at $24^{\circ} \mathrm{C}$ and $2.78 \mathrm{LOD}$ at $12^{\circ} \mathrm{C}$. The positions of the peak values for each QTL are shown in Table 3. Also the individual significance of each QTL, the proportion of variance explained by the QTL, and the additive effect of the QTL (i.e., the effect of the allele of the N2 strain compared to the effect of the allele of the CB4856 strain) are shown in this table.

QTL supporting genetic correlations within temperatures At $12^{\circ} \mathrm{C}$ we detected QTL located on chromosome $I V$ for egg size (peak at $-21.28 \mathrm{cM}$ ) and egg number (peak at $-9.96 \mathrm{cM})$. QTL for both traits had opposite effects, the additive effects were -3.64 for the QTL Qgg size $_{\text {and }} 11.38$ for the $\mathrm{QTL}_{\text {egg number, }}$ thus reflecting the negative genetic correlation between these traits. Although the peaks did not coincide at the same position, the confidence intervals of both QTL overlap and suggest a pleiotropic QTL according to Dupuis and Siegmund (1999), yet the possibility of closely linked genes in linkage disequilibrium cannot be ruled out.

QTL supporting genetic correlations across temperatures QTL for body mass colocated on chromosome $X$ for both temperatures (peaks at $-8.16 \mathrm{cM}$ ). Both QTL had a positive effect which supports the positive genetic 

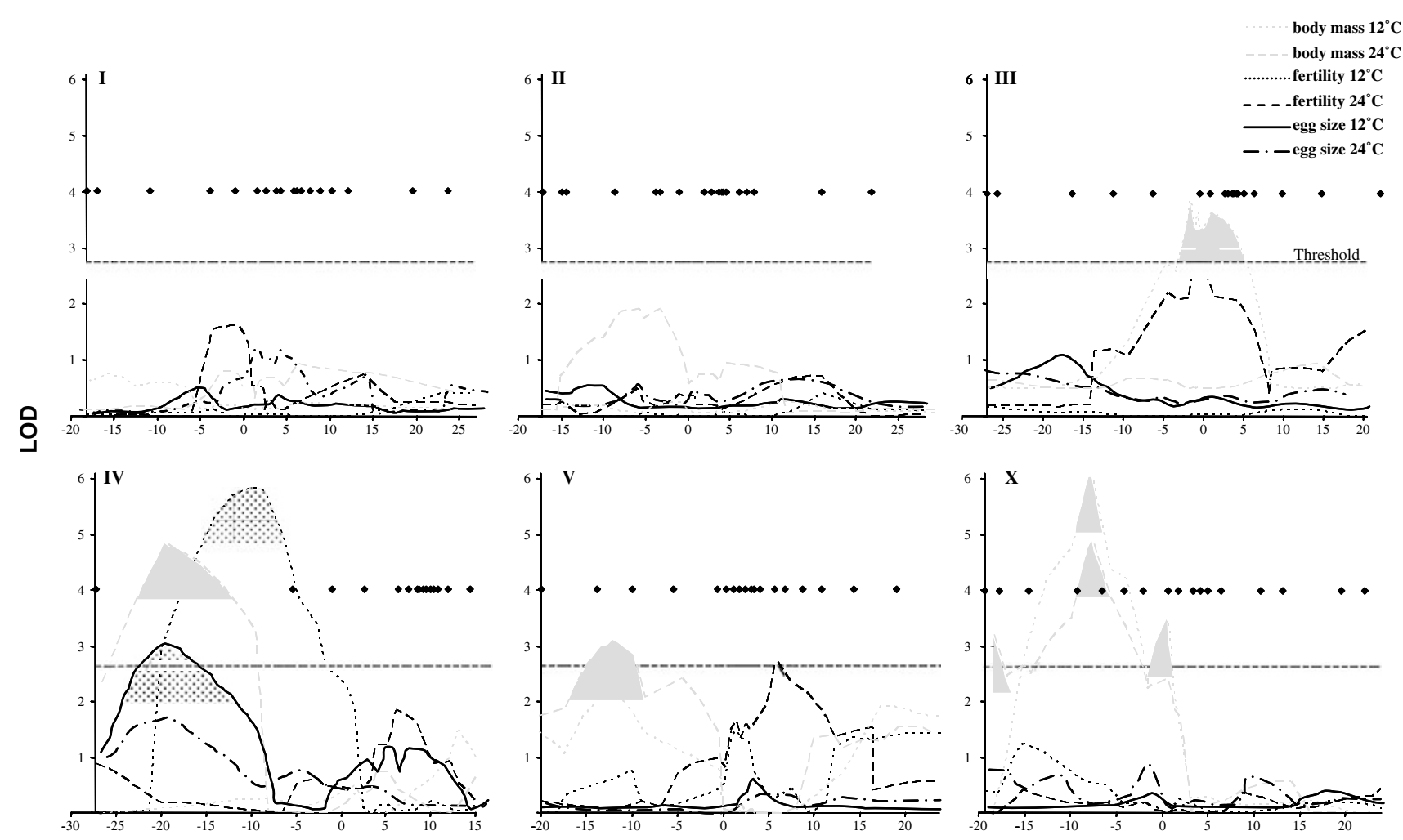

Genetic map position (cM)

Figure 1 QTL-mapping results for egg size and number and body mass in C. elegans at $24^{\circ} \mathrm{C}$ and $12^{\circ} \mathrm{C}$. QTL at chromosomes are shown with 1 LOD CI. Diamonds show the positions of the markers.

Table 3 QTL with maximum LOD scores for egg number, egg size and body mass at $24^{\circ} \mathrm{C}$ and $12^{\circ} \mathrm{C}$

\begin{tabular}{|c|c|c|c|c|c|c|}
\hline \multirow[t]{2}{*}{$\mathrm{T}$} & \multirow[t]{2}{*}{ trait } & \multicolumn{2}{|c|}{$Q T L$} & \multirow[t]{2}{*}{$\mathrm{R}^{2}$} & \multicolumn{2}{|c|}{ Effect } \\
\hline & & Map position & $L O D$ & & $a b s$ & $\%$ \\
\hline \multirow{5}{*}{$24^{\circ} \mathrm{C}$} & Egg number & V: 5.78 & $2.72^{*}$ & 0.12 & 11.3 & 6.38 \\
\hline & Body mass & IV: -19.96 & $4.89^{* * *}$ & 0.16 & -971.5 & -4.31 \\
\hline & & $\mathrm{V}:-12.09$ & $2.94^{*}$ & 0.09 & 751.0 & 3.33 \\
\hline & & X: -19.35 & $3.20^{*}$ & 0.11 & 836.7 & 3.71 \\
\hline & & $X:-8.16$ & $4.87^{* * *}$ & 0.16 & 1008.2 & 4.47 \\
\hline \multirow[t]{7}{*}{$12^{\circ} \mathrm{C}$} & Egg size & IV: -21.28 & $3.02 *$ & 0.15 & -0.5 & -3.64 \\
\hline & Egg number & IV: -9.96 & $5.84^{* * *}$ & 0.29 & 19.9 & 11.38 \\
\hline & Body mass & III: -1.39 & $3.87^{* *}$ & 0.12 & -867.2 & -3.49 \\
\hline & & III: -0.44 & $3.63^{* *}$ & 0.11 & -840.3 & -3.38 \\
\hline & & III: 1.42 & $3.62^{* *}$ & 0.11 & -837.8 & -3.37 \\
\hline & & $X:-8.16$ & $6.02^{* * *}$ & 0.21 & 1185.4 & 4.77 \\
\hline & & X: 0.42 & $3.46^{* *}$ & 0.13 & 943.4 & 3.80 \\
\hline
\end{tabular}

$R^{2}$ indicates the proportion of the among-strain variance explained by the QTL. Significance for LOD-scores $\left({ }^{*} P<0.05,{ }^{* *} P<0.01,{ }^{* * *} P<0.001\right)$ based on experiment-wise significance levels from 10,000 permutations. Absolute and relative effects as percentage of total s.d. of all RILs.

correlation found for this trait. QTL for egg number did not colocalize, a large QTL was found on chromosome $I V$ and a small, just significant, QTL on $V$. This agrees with the fact that we did not find a correlation across temperatures for this trait.

The QTL for body mass at $24^{\circ} \mathrm{C}$ on chromosome $I V$ (peak at $-19.96 \mathrm{cM}$ ) overlapped with QTL for egg size at $12^{\circ} \mathrm{C}$ (peak at 21, 28 cM) at chromosome $I V$. Both had negative effects which supports the positive correlation found between these traits across temperatures.

\section{Discussion}

We found significant genetic correlations between body mass, egg number and egg size at the two different temperatures. At $12^{\circ} \mathrm{C}$ we found a negative genetic correlation between egg size and number and at $24^{\circ} \mathrm{C}$, we found a positive correlation between egg size and body mass. The effect of temperature on genetic correlation has been reported for a number of life-history traits in different species. Windig (1994) studied the effect of 
different temperatures on the genetic correlation between developmental time, pupal weight and wing patterns in the butterfly Bicyclus aninana. The significant negative genetic correlation between developmental time and wing pattern found at $28^{\circ} \mathrm{C}$ changed to a positive correlation at $20^{\circ} \mathrm{C}$. Using selection experiments to study the effect of temperature on genetic correlations in Drosophila melanogaster, Norry and Loeschke (2002) also reported a reversal of genetic correlations between temperatures. These and our studies show that a significant correlation between traits at one temperature may not be detected at other temperatures. Therefore, evolutionary trajectories derived from genetic correlations depend on the ambient temperatures surrounding the organisms across generations (Sgrò and Hoffmann, 2004).

The evolutionary outcomes depend on the underlying mechanism of the genetic correlation, which can be studied by QTL analysis. In some cases, we were able to detect genomic regions associated with these correlations. The QTL analyses supported the negative correlation between egg size and number at $12^{\circ} \mathrm{C}$. Colocalizing QTL for both traits had opposite effects, the additive effects were -3.64 for the QTL $\mathrm{Q}_{\text {egg size }}$ and 11.38 for the QTL $_{\text {egg number }}$ These results suggest that the negative genetic correlation between these traits could be due to antagonistic pleiotropy, which was environment specific because we did not detect it at $24^{\circ} \mathrm{C}$. Across temperatures, the QTL for body mass at $24^{\circ} \mathrm{C}$ on chromosome $I V$ overlapped with QTL for egg size at $12^{\circ} \mathrm{C}$. This pleiotropic or closely linked QTL had the same direction of effect in both temperatures, thus supporting the positive correlation found between these traits across temperatures. Also across temperatures we found a positive correlation for body mass, which was supported by colocalizing QTL.

Genetic correlations between two traits become apparent when they are influenced by the same genes, or sets of genes. By reducing the degree to which traits in two environments can evolve independently (Via and Lande, 1985), such correlations may be important for the evolution of ecological specialization. Positive genetic correlations can accelerate the evolution of specific traits. Correlations between different traits in the same environment, or between the same trait in different environments, complicate the measurement of trait selection. In general, genetic correlations between traits due to pleiotropy can persist without selection, yet they can be shaped by natural selection acting on new mutations, which have variable effects on the correlation (Lande and Arnold, 1983).

The detection of only few significant QTL for egg size and number may be attributed to the relative small number of RILs (80) or the existence of many small insignificant QTL rather than a few major QTLs. Comparison with other genetically mapped traits with similar or low heritabilities suggests that it is unlikely that there was low power to detect multiple QTL because of low heritabilities.

Genetic correlations can be due to pleiotropy (a single locus affecting multiple traits), close linkage (association between loci due to physical proximity), linkage desequilibrium (statistical associations between loci due to evolutionary forces), or to the relationship with other, unrecorded traits. The maintenance of genetic correla- tions requires the existence of pleiotropic effects or very close linkage of genes. We found that the two QTL for egg size and number co-localized at $12^{\circ} \mathrm{C}$ and that the direction of the QTL effect were opposite thus supporting the negative genetic correlation. Colocalization of QTL may be the result of pleiotropy or close linkage. There are several ways to distinguish between these two alternatives. One is by statistical testing. For instance Lebreton et al. (1998) presented a nonparametric bootstrap method in which the RIL populations are multiply resampled and subsequent QTL CI values are used to evaluate the hypothesis of a single QTL affecting both traits. Another way to test for pleiotropy is by introgressing the genomic QTL region of interest into one genetic background. Measuring the focal trait would provide insight whether this region indeed affects that trait. This approach was adopted by Knight et al. (2001) for unraveling the underlying mechanism of a genetic tradeoff between body size and egg number in C. elegans. They found statistical evidence for a pleiotropic QTL, but this was not subsequently supported by the phenotypic measurements using introgression strains, since the existence of one QTL affecting both traits could not be detected. Instead they found more than one region affecting each trait separately. Another reason for their findings may be that undetected epistatic interactions had affected the QTL in the RILs. Once such a region were introgressed in isolation from these epistatic interacting loci, the phenotype would no longer be detected. However it should be realized that QTL mapping is limited to the detection of genomic regions affecting a trait and it is difficult to detect single loci (Hausmann et al., 2005). A possible solution would be to use denser genetic maps allowing for fine-scale mapping, perhaps in combination with crossing experiments between selected introgression strains.

Negative correlations between egg size and number have been observed before for many species (Guisande et al., 1996; Christians, 2000; Brown et al., 2003) and can be explained as an optimization of both number and size of offspring in order to maximize fitness. However, Zera and Harshman (2001) highlighted the limitations of finding genetic correlations without an understanding of the pysiological mechanisms underlying the trade-off. A promising way forward may be to study potential pleiotropically acting genes at the mRNA level. For example, Bochdanovits and de Jong (2004) analyzed natural populations of Drosophila melanogaster for the molecular mechanisms underlying trade-offs. They analyzed pre-adult survival and adult body weight and detected 34 genes suggesting either a 'low weight, high survival' or 'high weight, low survival' trade-off on the expression level.

Mapping life-history traits in C. elegans has been performed before (Johnson and Hutchinson, 1993; Shook et al., 1996; Shook and Johnson, 1999; Knight et al., 2001) but, due to the type of markers used, these studies did not analyze the whole genome, accordingly reducing the power for detecting a (pleiotropic or closely linked) QTL. Besides, the inbred-panel approach suffers from low vitality because of deleterious effects of transposon markers (originating from the the parental strain BOBergerac) (Hodgkin and Doniach, 1997), which might have obscured or biased the results due to selection for N2 genes. We used equally viable (Hodgkin and 
Doniach, 1997) strains for which a large number of SNP have been characterized genome-wide thus enabling a more complete molecular genetic analysis of the intercrossed strains.

Despite the fact that we found significant correlations, which were supported by QTL for each environment, we could not reject the hypothesis that correlations were different between environments based on parametric tests. In this study all analyses of genetic correlations were based on RIL means. An alternative method would involve estimation of genetic correlations from covariance components. Both approaches suffer from particular drawbacks (e.g. possible bias in the first method resulting from neglecting within-RIL variances (Astles et al., 2006)). Therefore, we decided to perform additional analysis, in which we compared genetic variancecovariance matrices of the three investigated life-history traits between the temperatures. For that purpose we used Flury hierarchy approach (Flury, 1988) as implemented by Phillips for the purposes of quantitative genetics (Phillips and Arnold, 1999). In this approach a series of hypotheses regarding different levels of similarity between the matrices is tested (from unrelated structure to equality). The tests involve comparisons of the principal components (eigenvectors) as well as eigenvalues. The results obtained with the CPCrand software (Phillips, 1998) indicated that there was insufficient evidence for rejection of the hypothesis of equality of the genetic matrices in $12^{\circ} \mathrm{C}$ and $24^{\circ} \mathrm{C}(\mathrm{CPC} 1 ; P=0.35)$. There is therefore no discrepancy in the results obtained with the parametric test. It should be noted that the genetic component of variance for RILs is treated in CPCrand software as half of the among-RIL variance, which is different from the broad-sense genetic variance, which we utilized in RILs' means approach. Another factor that may have influenced the results is the power to detect differences in direction of matrix eigenvectors which is considered to be low for sample sizes that are commonly used in such studies (Mezey and Houle, 2003).

We found different genetic correlations at different temperatures in $C$. elegans and we have been able to detect loci involved in the control of these correlations. We found evidence for possible pleiotropic or closely linked effects, which implies that these correlations have evolutionary significance. Whether these mapped loci also control correlations under field conditions in natural populations is hard to say because the experienced temperatures in soils are likely to be lower than $12^{\circ} \mathrm{C}$. However our results provide evidence for a dynamic genetic control of life-history correlates.

\section{Acknowledgements}

We thank the Caenorhabditis Genetics Center (St Paul, Minnesota) for providing the nematode strains Bristol N2 and CB4856 and A Mucha for practical assistance in breeding the RILs. EWG and JAGR were financed by the Netherlands Organization for Scientific Research (NWO Grant no. 925-01-026).

\section{References}

Astles PA, Moore AJ, Preziosi RF (2006). A comparison of methods to estimate cross-environment genetic correlations. J Evol Biol 19: 114-122.
Ayyadevara S, Ayyadevara R, Vertinob A, Galeckic A, Thadena JJ, Shmookler Reis RJ (2003). Genetic loci modulating fitness and life span in Caenorhabditis elegans: categorical trait interval mapping in CL2a $\times$ Bergerac-BO recombinant-inbred worms. Genetics 163: 557-570.

Bochdanovits Z, de Jong G (2004). Antagonistic pleiotropy for life-history traits at the gene expression level. Proc $R$ Soc Lond Ser B-Biol Sci 271: S75-S78.

Brown CA, Sandford BM, Swerdon RR (2003). Clutch size and offspring size in the wolf spider Pirata sedentarius (Araneae, Lycosidae). J Arachnol 31: 285-296.

Byerly L, Cassada RC, Russell RL (1976). The life cycle of the nematode Caenorhabditis elegans. Develop Biol 51: 23-33.

Christians JK (2000). Trade-offs between egg size and number in waterfowl: an interspecific test of the van Noordwijk and de Jong model. Funct Ecol 14: 497-501.

Churchill GA, Doerge RW (1994). Empirical threshold values for quantitative trait mapping. Genetics 138: 963-971.

Czesak ME, Fox C (2003). Evolutionary ecology of egg size and number in a seed beetle: genetic trade-offs differ between environments. Evolution 57: 1121-1132.

Dupuis J, Siegmund D (1999). Statistical methods for mapping quantitative trait loci from a dense set of markers. Genetics 151: 373-386.

Emmons SW, Klass MR, Hirsch D (1979). Analysis of the constancy of DNA sequences during development and evolution of the nematode Caenorhabditis elegans. Proc Natl Acad Sci USA 76: 1333-1337.

Flury B (1988). Common Principal Components and Related Multivariate Models. Wiley: New York.

Fox CW (1997). Egg-size manipulations in the seed beetle Stator limbatus: consequences for progeny growth. Can J Zool 75: 1465-1473.

Gems D, Sutton AJ, Sundermeyer ML, Albert PS, King KV, Edgleya ML et al. (1998). Two pleiotropic classes of daf-2 mutation affect larval arrest, adult behavior, reproduction and longevity in Caenorhabditis elegans. Genetics 150: 129-155.

Guisande C, Sánchez J, Maneiro I, Miranda A (1996). Trade-off between offspring number and offspring size in the marine copepod Euterpina acutifrons at different food concentrations. Mar Ecol Prog Ser 143: 37-44.

Gutteling EW, Riksen JG, Bakker J, Kammenga JE (2007). Mapping phenotypic plasticity and genotype-environment interactions affecting life history traits in Caenorhabditis elegans. Heredity 98: 28-37.

Hausmann NJ, Juenger TE, Sen S, Stowe KA, Dawson TE, Simms EL (2005). Quantitative trait loci affecting $\delta^{13} \mathrm{C}$ and response to differential water availibility in Arabidopsis thaliana. Evolution 59: 81-96.

Hekimi S, Lakowski B, Barnes TM, Ewbank JJ (1998). Molecular genetics of life span in C. elegans: how much does it teach us? Trends Genet 14: 14-20.

Hodgkin J, Doniach T (1997). Natural variation and copulatory plug formation in Caenorhabditis elegans. Genetics 146: 149-164.

Johnson TE, Hutchinson EW (1993). Absence of strong heterosis for life-span and other life-history traits in Caenorhabditis elegans. Genetics 134: 465-474.

Juenger T, Pérez-Pérez JM, Bernal S, Micol JL (2005). Quantitative trait loci mapping of floral and leaf morphology traits in Arabidopsis thaliana: evidence for modular genetic architecture. Evol Develop 7: 259-271.

Kenyon C, Chang J, Gensch E, Rudner A, Tabtiang R (1993). A C. elegans mutant that lives twice as long as wild-type. Nature 366: 461-464.

Knight CG, Azevedo RBR, Leroi AM (2001). Testing lifehistory pleiotropy in Caenorhabditis elegans. Evolution 55: 1795-1804.

Lande R, Arnold SJ (1983). The measurement of selection on correlated characters. Evolution 37: 1210-1226. 
Lebreton CM, Visscher PM, Haley CS, Semikhodskii A, Quarrie SA (1998). A nonparametric bootstrap method for testing close linkage vs pleiotropy of coincident quantitative trait loci. Genetics 150: 931-943.

Lewis JA, Fleming JT (1995). Basic culture methods, In: Epstein HF, Shakes DC (eds). Caenorhabditis elegans: Modern Biological Analysis of an Organism. Academic Press, Inc.: San Diego, California. pp 3-29.

Li Y, Alvarez OA, Gutteling EW, Tijsterman M, Fu J, Riksen JAG et al. (2006). Mapping determinants of gene expression plasticity by genetical genomics in C. elegans. PloS Genet, doi:10.1371/journal.pgen.0020222.eor.

Mezey JG, Houle D (2003). Comparing G matrices: are common principal components informative? Genetics 165: 411-425.

Norry FM, Loeschke VR (2002). Longevity and resistance to cold stress in cold-stress selected lines and their controls in Drosophila melanogaster. J Evol Biol 15: 775-783.

Phillips PC (1998). Designing experiments to maximize the power of detecting correlations. Evolution 52: 251-255.

Phillips PC, Arnold SJ (1999). Hierarchical comparison of genetic variance-covariance matrices. I. Using the flury hierarchy. Evolution 53: 1506-1515.

Roff DA (2000). Trade-offs between growth and reproduction: an analysis of the quantitative genetic evidence. J Evol Biol 13: $434-445$.

SAS Institute (2003). SAS Software, version 9.1, Cary: NC, USA. Sokal RR, Rohlf FJ (1995). Biometry. Freeman: San Francisco.

Sgrò CM, Hoffmann AA (2004). Genetic correlations, tradeoffs and environmental variation. Heredity 93: 241-248.

Shook DR, Brooks A, Johnson TE (1996). Mapping quantitative trait loci affecting life history traits in the nematode Caenorhabditis elegans. Genetics 142: 801-817.
Shook DR, Johnson TE (1999). Quantitative trait loci affecting survival and fertility-related traits in Caenorhabditis elegans show genotype-environment interactions, pleiotropy and epistasis. Genetics 153: 1233-1243.

Stearns SC (1992). The Evolution of Life Histories. Oxford University Press: New York.

Van Voorhies WA (1996). Bergmann size clines: a simple explanation for their occurrence in ectotherms. Evolution 50: 1259-1264.

Vaughton G, Ramsey M (1998). Sources and consequences of seed mass variation in Banksia marginasa (Proteaceae). J Ecol 86: 563-573.

Via S, Lande R (1985). Genotype-environment interactions and the evolution of phenotypic plasticity. Evolution 39: 505-522.

Wang S, Basten CJ, Zeng Z-B (2001). Windows QTL Cartographer 2.0 , pp. Department of Statistics, North Carolina State University: Raleigh, NC (http://statgen.ncsu.edu/qtlcart/ WQTLCart.htm).

Wang DL, Zhu J, Li ZK, Paterson AH (1999). Mapping QTLKs with epistatic effects and QTLxenvironment interactions by mixed linear models appraoches. Theor Appl Genet 99: 1255-1264.

Williams GC (1957). Pleiotropy, natural-selection and the evolution of senescence. Evolution 11: 398-411.

Windig JJ (1994). Reaction norms and the genetic basis of phenotypic plasticity in the wing pattern of the butterfly Bicyclus anynana. J Evol Biol 7: 665-695.

Winkler DW, Wallin K (1987). Offspring size and number: a life history model linking effort per offspring and total effort. Am Nat 129: 708-720.

Zera AJ, Harshman LG (2001). The physiology of life history trade offs in animals. Ann Rev Ecol Syst 32: 95-126. 